\title{
INFLUENCIA DE LA INDUSTRIA DEL PETRÓLEO DEL DEPARTAMENTO DE SANTANDER EN SU SISTEMA REGIONAL DE INNOVACIÓN (SRI): UN ANÁLISIS EN COLOMBIA
}

\section{THE INFLUENCE OF SANTANDER'S OIL INDUSTRY ON ITS REGIONAL INNOVATION SYSTEM (RIS): AN ANALYSIS IN COLOMBIA}

\author{
Cinthya Carolina Arias Manjarrez ${ }^{1}$ \\ Piedad Arenas Díaz ${ }^{2}$ \\ Jaime Alberto Camacho Pico ${ }^{3}$
}

\section{RESUMEN}

La regionalización de la ciencia, tecnología e innovación (CTI) es una de las políticas de desarrollo económico y social de Colombia, siendo una de las razones por las que los territorios se han incrementado los esfuerzos en el fortalecimiento de los sistemas regionales de innovación (SRI) como Sucede en Santander. Esta investigación se centra en el estudio de la influencia de la industria petrolera en el SRI, para identificar el papel de la industria en el desarrollo y consolidación de estos sistemas. La metodología de investigación se dividió en tres etapas: la caracterización de actores y redes, la medición de la influencia científica y tecnológica en el sistema y medir la influencia en otro sector a través de los indicadores de CTI. Se identificó que la industria ha influido en la formación de recursos humanos, producción de patentes y publicaciones, consolidando la capacidad de investigación y desarrollo, y la cooperación regional. Contribuyó a romper la integración teórica de las principales características de los actores de la ISR y para responder a la pregunta: ¿Cuál ha sido la influencia de las industrias en el SRI? Proporcionar una metodología replicable. Y una contribución práctica en la identificación de acciones de la industria que contribuyen a la consolidación de la ISR, que puede ser mejorado y replicado en otros sectores y regiones.

Palabras clave: Sistema Regional de Innovación. La industria petrolera. Indicadores de CTI. Santander.

\footnotetext{
ABSTRACT

Regionalization of science, technology and innovation is one of the policies to promote economic and social development in Colombia, whereby territories have increased their efforts in strengthening regional innovation systems (RIS). This is the case of Santander. In order to identify the role of industries in development and consolidation of these systems, this research studies the influence of the oil industry in its RIS. This work wasdone in three stages: characterization of actors and networks, measurement of scientific and technological influence on the system and measurement of it in another industry using STI indicators. We found that this industry has influenced on formation of human resources, patents and publications production, capacity building to $\mathrm{R} \& \mathrm{D}$ and regional cooperation. From a theoretical approach, it contributes to integrate of the main characteristics of actors in RIS and to provide an answer to the question What has been the influence of the industries on the RIS? Supplying a replicable

${ }^{1}$ Universidad Industrial de Santander - Colômbia. Magíster en Política y Gestión de la Ciencia y la tecnología en INNOTEC, Escuela de Estudios Industriales y Empresariales. Bucaramanga, Carrera 27, calle 9, Colômbia. E-mail: parenasd@uis.edu.co

${ }^{2}$ Universidad Industrial de Santander, Colômbia. Candidata a magíster em Ingeniería Industrial en INNOTEC, Escuela de Estudios Industriales y Empresariales. Bucaramanga, Carrera 27, calle 9, Colômbia. E-mail: cinthya.arias@ correo.uis.edu.co

${ }^{3}$ Universidad Industrial de Santander, Colômbia. Doctor en Ingeniería Industrial en INNOTEC, Escuela de Estudios Industriales y

Empresariales. Bucaramanga, Carrera 27, calle 9, Colômbia. E-mail: jcamacho@uis.edu.co
} 
methodology. From a practical approach, it contributes to identify industry's actions that contribute to strengthening the RIS, which may be potentiated and replicated in other sectors and regions.

Keywords: Regional Innovation System. Oil industry. STI Indicators. Santander.

\section{Introducción}

Existe un acuerdo, casi generalizado en la literatura académica, que el conocimiento, el aprendizaje y la innovación son fundamentales para el desarrollo económico y la competitividad de las empresas, las regiones y las naciones (TÖDTLING; TRIPPL, 2005), de ahí que en los últimos años surgieran diferentes enfoques teóricos que pretenden explicar tal relación. Uno de estos, es el de sistemas regionales de innovación (SRI), definido por Cooke (2003) como un entramado constituido por subsistemas de generación y explotación de conocimiento que interactúan y se encuentran vinculados a otros sistemas regionales, nacionales y globales, para la comercialización de nuevo conocimiento. Desde su mención en la literatura numerosas investigaciones han abordado su estudio, lo cual se refleja en el incremento anual de la producción científica en el área; algunas de las principales causas de éste crecimiento están dadas:

1) por la necesidad de evitar la distorsión y la pérdida de la información bajo la hipótesis de los sistemas nacionales como entidades homogéneas (MORGAN, 2004), ya que el patrón altamente desigual y la difusión de la innovación en el espacio podría estar mejor representado asumiendo unidades sub-nacionales de análisis;

2) por el creciente interés por la innovación como fuente de ventaja competitiva (COOKE, 1995);

3) porque las competencias y las instituciones políticas están parcialmente ligados a territorios subnacionales (COOKE; BOEKHOLT; TÖDTLING, 2000);

4) por la necesidad de nuevas políticas para hacer frente a las desigualdades regionales y a la divergencia (ASHEIM; LAWTON; OUGHTON, 2011); y

5) porque la especialización industrial y los resultados de la innovación, difieren entre las regiones (BRESCHI, 2000; HOWELLS, 1999).

Con el propósito de comprender el comportamiento y características diferenciadoras de cada sistemas de innovación, se han propuesto diferentes modelos para abordar el mismo tomado como referencia la capacidad regional de innovación (DOLOUREX; PARTO, 2005) (FURMAN; PORTER; STERN, 2002). En la literatura 
se presentan algunos modelos que pretenden explicarla a diferentes escalas geográficas y más aún, la importancia de que ésta sea evaluada de forma integral. Esta necesidad de definir y medir con indicadores no sólo tiene como objetivo mejorar la representación de los sistemas de Innovación, sino también profundizar en sus características particulares (ITURRIAGAGOITIA; SAEZ; GRACIA, 2005), de tal manera que una región específica reconozca sus ventajas y desventajas, y pueda diseñar políticas efectivas para mejorar su capacidad de innovación (LI, 2011). En efecto, la evaluación de los SRI es uno de los temas más discutidos en este campo de la investigación (IAMMARINO, 2005) considerando que, dado que las características de la red de innovación difieren entre los entornos regionales (YUN; LEE, 2013), no se podría hablar de un único modelo de medición estándar. De esta forma, el acuerdo implícito de la existencia un vacío sobre los indicadores adecuados para medir I+D y la innovación tecnológica (DEN HERTOG; ROELANDT; BOEKHOLT, 1995; INZELT, 2004; WANGNER-DÖBLER，2005; ITURRIAGAGOITIA，SAEZ; GRACIA，2005), ha hecho que los modelos de medición tengan profundas divergencias, por los determinantes que usan, y que los resultados entre ellos no sean comparables.

De otra parte, en la definición misma de SRI se denota la importancia de los actores que lo conforman y de sus interacciones, por eso los agentes del SRI son la variable clave en los análisis de los SRI (DOLOREUX; PARTO, 2005), de ahí la necesidad de definir el papel de cada uno de ellos y porque, consecuentemente, el desempeño innovador de una economía dependerá de la capacidad de innovación de sus empresas, centros de investigación y sus relaciones con otras instituciones (DOLOREUX, 2002). Por consiguiente, en la literatura se encuentran diferentes modelos que explican el funcionamiento del sistema de innovación a partir de la configuración económica y socio-cultural de los actores que lo constituyen (AUTIO, 1998; TRIPPL; TÖDTLING, 2007; MENÉNDEZ; DE LUCIO; JIMÉNEZ, 2011). En ellos se destaca el papel de las aglomeraciones industriales, las cuales son consideradas tan importantes en la literatura de SI que algunos autores, como Doloreux y Parto (2005), Bjørn T. Asheim e Isaksen (1996), Asheim y Isaksen (2002) y Cooke (1997), han descrito el sistema como uno en el que una estructura de producción está incluida en una estructura institucional en el que las empresas y otras organizaciones se dedican sistemáticamente al aprendizaje interactivo o como clústeres regionales que son apoyados por instituciones y organizaciones del entorno. 
Reconociendo la importancia del sistema productivo en los SRI, la presente investigación aborda el caso de la industria del petróleo del departamento de Santander/Colombia, mostrando, de forma empírica, como el SRI ha sido influenciado por esta industria y específicamente cómo otras industrias de la región han estado vinculadas a los productos científicos-tecnológicos de la primera, con lo cual se contribuirá a dar una repuesta, usando indicadores de ciencia, tecnología e innovación (CTI), a la pregunta ¿Cuál ha sido la influencia de las industrias en los SRI?, proporcionando, además, un diseño metodológico que puede ser replicado en otras industrias y por otros actores. Para ello, se realiza una caracterización de los principales actores departamentales de la industria del petróleo, un análisis de la participación de la actividad petrolera sobre los indicadores de innovación del departamento de Santander, y la identificación de la influencia de la industria del petróleo en el desarrollo de capacidades de innovación en otras industrias de la región. De esta forma, el trabajo se estructura en tres partes: metodología, en la que se dan a conocer las etapas seguidas en la investigación; resultados, donde se describe el papel de la industria del petróleo en el SRI; y conclusiones, donde se evidencian los aspectos más importantes identificados en el estudio.

\section{Metodología}

La metodología utilizada en el desarrollo de la investigación se realizó en tres fases: caracterización de actores, identificación de la influencia de la industria del petróleo en el Sistema Regional de Innovación de Santander y la identificación de la influencia de la industria del petróleo en la otra industria de la región.

\subsection{Caracterización de los actores}

En esta fase se describen los actores pertenecientes tanto al SRI del departamento de Santander a través de una revisión documental de los estudios previos del SRI como al Clúster del petróleo y gas, que agrupa los principales agentes de la industria del petróleo de la región, los cuales fueron caracterizados de acuerdo a los principales criterios encontrados en la literatura. Posteriormente se realizó un estudio de las de redes con el fin recolectar evidencia de las relaciones entre los actores identificados. Se efectuó una revisión del banco de iniciativas relacionadas con la CTI en la región (ARIAS; ARENAS, 2013) y las páginas web de los actores fueron consultadas para identificar: actividades conjuntas y alianzas con otros. 
Consecutivamente, se valoró la intensidad de las relaciones identificadas en una escala de 0 a 4, donde la primera representaba la ausencia de relaciones y la última una alta intensidad de la misma. Después, utilizando el software Lipsor Mactor ${ }^{\circledR}$, se valoraron la relaciones de los actores, para determinar los agentes articuladores del sistema que pertenecen a la industria del petróleo y se realizó una evaluación de la posición de cada uno ellos frente a las estrategias de articulación de los actores del sistema nacional de ciencia, tecnología e innovación-SNCTI determinadas por Monroy (2006): cofinanciar proyectos, realizar eventos de integración, financiar proyectos conjuntos, fortalecer grupos y redes, crear espacios de interacción, incrementar convenios de cooperación, apoyar formación de capital humano, establecer mecanismos de coordinación presupuestal y alinear las necesidades con la oferta de servicios. Con el uso del software mencionado se obtuvieron los resultados de las redes y la cercanía de los actores por estrategia.

\subsection{Identificación de la influencia de la industria del petróleo en el Sistema Regional de Innovación de Santander}

Se realizó una revisión de los modelos de medición de innovación que han usado algunas regiones y países, igualmente usado por el OCyT para el análisis científico tecnológico en las regiones colombianas. De esos se identificaron las dimensiones y variables de medición de la innovación comunes y los indicadores que permitieran caracterizar cada una de variables de acuerdo con lo expuesto en los modelos y con la información disponible de cada una de ellas. Posteriormente se estudió el sistema regional de innovación a través de indicadores de ciencia, tecnología e innovación (ARIAS; ARENAS; FLÓREZ; CARRILLO, 2013) y finalmente se identificó la participación de las actividades de la industria del petróleo por cada indicador, descartando aquellos en los que, por definición, la industria no podría tener influencia y en los que no se conoce la línea base. Para ello se realizaron las siguientes actividades de acuerdo a las principales variables, que son: Capital humano, Producción científica, Sistema de investigación, Efectos económicos. Para el primero se tomó como referencia la información disponible de los graduados en áreas afines; para el segundo se analizó la producción de artículos científicos en el área según datos ISI Web of Knowledge apoyándose del software Vantage Point ${ }^{\circledR}$ y de patentes según las registradas en la Organización Mundial de la Propiedad Intelectual - OMPI y en la United States Patent and Trademark Office - UPSTO empleando el software Matheo Patent ${ }^{\circledR}$. En la 
caracterización de la participación en el Sistema de Investigación, se tomó como referencia la información proporcionada por COLCIENCIAS en la clasificación de grupos de investigación del año 2013 y finalmente, para la última variable se recolectó información de fuentes secundarias asociadas a cada uno de los indicadores.

\subsection{Identificación de la influencia de la industria del petróleo en la otra industria de la región}

En esta fase se realizó una revisión de la cadena productiva del petróleo y gas para identificar otras industrias vinculadas y, se seleccionó la industria a estudiar según su comportamiento científico-tecnológico en el departamento. Posteriormente se determinaron los tipos de influencias científico-tecnológicas que la industria del petróleo puede generar en la otra, según la metodología diseñada por Sarmiento, Jaime, Arenas, Becerra, y Camacho (2011), que tiene como principales determinantes:

a) Formación de recursos humanos en nuevas tecnologías y gestión tecnológica.

b) Registro y homologación de patentes.

c) Registro y documentación técnica del know-how.

d) Desarrollo de capacidades de diseño e ingeniería en la entidad o grupo.

e) Consolidación de capacidades para realizar actividades de I\&D:

- creación o fortalecimiento de Grupos de I\&D;

- dotación de laboratorios de I\&D o de calidad y plantas piloto;

- redes de información y colaboración científico tecnológica.

f) Mejoramiento en la oferta y pertinencia de los servicios tecnológicos.

g) Publicaciones, cooperación regional e internacional.

Posteriormente se recolectó la información asociada a cada tipo de influencia, para lo cual se hizo uso de los software Matheo Patent® para el análisis de patentes y Vantage Point ${ }^{\circledR}$ para los artículos científicos. Se realizaron búsquedas en la base de datos ISI WEB OF SCIENCE, en la plataforma ScienTI de Colciencias, en la plataforma SNIES del Ministerio de Educación Nacional, en las bases de datos de las Cámaras de Comercio de la región, en las estadísticas del DANE, en la plataforma de indicadores de competitividad DNP, en páginas web de los actores involucrados, entre otros. 


\section{Resultados}

De acuerdo a cada una de las fases metodológicas realizadas en la investigación, se presentan los resultados obtenidos.

\subsection{Caracterización de actores}

El estudio de los actores de los SRI es un tema en revisión permanente; sin embargo, en la literatura se evidencia características asociadas a cada uno de los tipos de agentes que participan en el entramado que se conoce como sistema de innovación. Para efectos del estudio, en la Tabla 1 se exponen algunos criterios relevantes expuestos por diferentes autores para la caracterización de los de los agentes del sistema.

Los actores pertenecientes al SRIS y Clúster del Gas y del Petróleo (CPG) son 34 de los 104 que conforman el SRI del Departamento de Santander. En la composición por subsistemas, predominan los actores pertenecientes al entorno académico-científico con un $67 \%$ de participación, facilitador $15 \%$, productivo y tecnológico $9 \%$ cada uno y no hay presencia de actores del subsistema financiero. El 76,5\% de los actores son entidades privadas, de los cuales el $69 \%$ corresponden a entidades científicoacadémicas, seguidas de actores tecnológicos, productivos y facilitadores que representan el $12 \%, 11 \%$ y $8 \%$ respectivamente. De forma similar, del 23,5\% de las entidades públicas, la mayoría (62\%) son entidades científico-académicas y el $38 \%$ son facilitadoras.

Los actores pertenecientes al SRIS y Clúster del Gas y del Petróleo (CPG) son 34 de los 104 que conforman el SRI del Departamento de Santander. En la composición por subsistemas, predominan los actores pertenecientes al entorno académico-científico con un $67 \%$ de participación, facilitador $15 \%$, productivo y tecnológico $9 \%$ cada uno y no hay presencia de actores del subsistema financiero. El 76,5\% de los actores son entidades privadas, de los cuales el $69 \%$ corresponden a entidades científicoacadémicas, seguidas de actores tecnológicos, productivos y facilitadores que representan el $12 \%, 11 \%$ y $8 \%$ respectivamente. De forma similar, del 23,5\% de las entidades públicas, la mayoría (62\%) son entidades científico-académicas y el $38 \%$ son facilitadoras. 
Tabla 1 - Categorización de actores de los sistemas regiones de innovación

\begin{tabular}{|c|c|c|c|c|c|c|c|}
\hline \multicolumn{7}{|c|}{ ESPECIFICIDAD DEL PROPÓSITO } & SECTOR \\
\hline \multirow{3}{*}{\multicolumn{2}{|c|}{ Organizaciones }} & \multirow{2}{*}{\multicolumn{3}{|c|}{$\begin{array}{l}\text { Productor de conocimiento } \\
\text { Distribuidor de conocimiento }\end{array}$}} & \multirow{3}{*}{ Instituciones } & \multirow{2}{*}{\begin{tabular}{|l|} 
Formales \\
Informales
\end{tabular}} & \multirow[t]{2}{*}{ Público } \\
\hline & & & & & & & \\
\hline & & \multicolumn{3}{|c|}{ Regulador de conocimiento } & & $\begin{array}{l}\text { Básicas } \\
\text { De soporte }\end{array}$ & Privado \\
\hline \multicolumn{8}{|c|}{ SUBSISTEMAS SEGÚN FUNCIÓN } \\
\hline \multirow{5}{*}{ Productivo } & \multicolumn{2}{|c|}{ Empresa } & \multirow{7}{*}{ Tecnológico } & \multicolumn{2}{|c|}{$\begin{array}{l}\text { Parques } \\
\text { Tecnológicos }\end{array}$} & \multicolumn{2}{|r|}{$\begin{array}{l}\text { Fondos capital } \\
\text { riesgo }\end{array}$} \\
\hline & \multirow{2}{*}{\multicolumn{2}{|c|}{$\begin{array}{l}\text { Asociaciones } \\
\text { Empresariales }\end{array}$}} & & \multirow{2}{*}{\multicolumn{2}{|c|}{$\begin{array}{l}\text { Incubadoras de } \\
\text { empresas }\end{array}$}} & \multirow{4}{*}{ Financiero } & $\begin{array}{l}\text { Fondos } \\
\text { internacionales }\end{array}$ \\
\hline & & & & & & & Cooperativas \\
\hline & \multirow{2}{*}{\multicolumn{2}{|c|}{$\begin{array}{l}\text { Cámaras de } \\
\text { comercio }\end{array}$}} & & \multirow{2}{*}{\multicolumn{2}{|c|}{$\begin{array}{l}\text { Centros de } \\
\text { desarrollo } \\
\text { Productivo }\end{array}$}} & & Cofinanciadores \\
\hline & & & & & & & Banca \\
\hline \multirow{2}{*}{$\begin{array}{l}\text { Científico- } \\
\text { académico }\end{array}$} & \multicolumn{2}{|c|}{ IES } & & \multicolumn{2}{|c|}{$\begin{array}{l}\text { Centros de } \\
\text { desarrollo } \\
\text { tecnológicos }\end{array}$} & \multirow[b]{2}{*}{ Facilitador } & $\begin{array}{l}\text { Agencias de } \\
\text { desarrollo } \\
\text { regional }\end{array}$ \\
\hline & \multicolumn{2}{|c|}{$\begin{array}{l}\text { Centros de } \\
\text { investigación }\end{array}$} & & \multicolumn{2}{|c|}{$\begin{array}{l}\text { Oficinas de } \\
\text { Transferencia de } \\
\text { Resultados de } \\
\text { Investigación }\end{array}$} & & $\begin{array}{l}\text { Actores } \\
\text { gubernamentales }\end{array}$ \\
\hline
\end{tabular}

Fuente: adaptada de Lucio y Castro (1995), Edquist (1997), Tödtling y Trippl (2005), Monroy (2006), y Arias, Arenas, Flórez y Carrillo (2013).

Del entorno cientifico-académico, el $83 \%$ son instituciones de educacion superior, $13 \%$ centros de investigación y $4 \%$ centros de formacion avanzada. De las IES, las unversidades tienen mayor presencia con el 35\% del total, seguidas de las intituciones universitarias con un $31 \%$ de participación en el total. El subsistema facilitador, comprende agencias de desarrollo regional e instituciones gubernamentales, que representan el $40 \%$ y $60 \%$ respectivamente. La industria en el sistema en estudio tiene un parque tecnológico, una OTRI y un Centro de Desarrollo tecnológico (CDT), quienes componen el subsistema tecnológico mientras que el sistema productivo está conformado por dos cámaras de comercio y el conjunto de las empresas. Este último, aunque es considerado un solo actor por efecto del estudio, representa, al menos, a las 446 empresas pertenecientes al Clúster del Petróleo y Gas.

Por otra parte, del total de los actores del SRI y CPG el $21 \%$ corresponde a instituciones y el $79 \%$ a organizaciones. Más de la mitad de estas instituciones son formales y de soporte, es decir, influyen en un sector específico. En cuanto a las organizaciones, 24 son generadoras de conocimiento y tres realizan difusión y adaptación de conocimiento, entre las que se encuentra el Parque Tecnológico Guatiguará, que cuenta en sus áreas estratégicas, con la de energía.

El análisis de las relaciones, permitió diferenciar los actores de enlace que satisfacen la definición de SRI del "entramado de actores" que realmente establece las principales relaciones en el sistema de innovación. De esta forma se determinó que diez de los actores no tienen enlaces con otros agentes, de los cuales nueve hacen parte del 
subsistema científico y son, en su mayoría, instituciones de educación superior, lo que implica que al apegarse a los requisitos del SRI, este subsistema reduciría su participación en el sistema un $13 \%$. Del mapa de la figura 1, se destaca la proximidad entre los actores del subsistema facilitador, como los que más posiciones comunes tienen ante las ocho estrategias para la articulación del sistema nacional de ciencia, tecnología e innovación, lo que se considera beneficioso ya que son ellos los encargados de servir de plataforma y de velar por la articulación de los actores del sistema. Seguida de estos, la próxima convergencia más clara es la de las universidades las cuales, al cumplir funciones misionales similares, participan en las estrategias de forma alineada.

Figura 1 - Convergencia de los actores según tipo de estrategias en la que participan

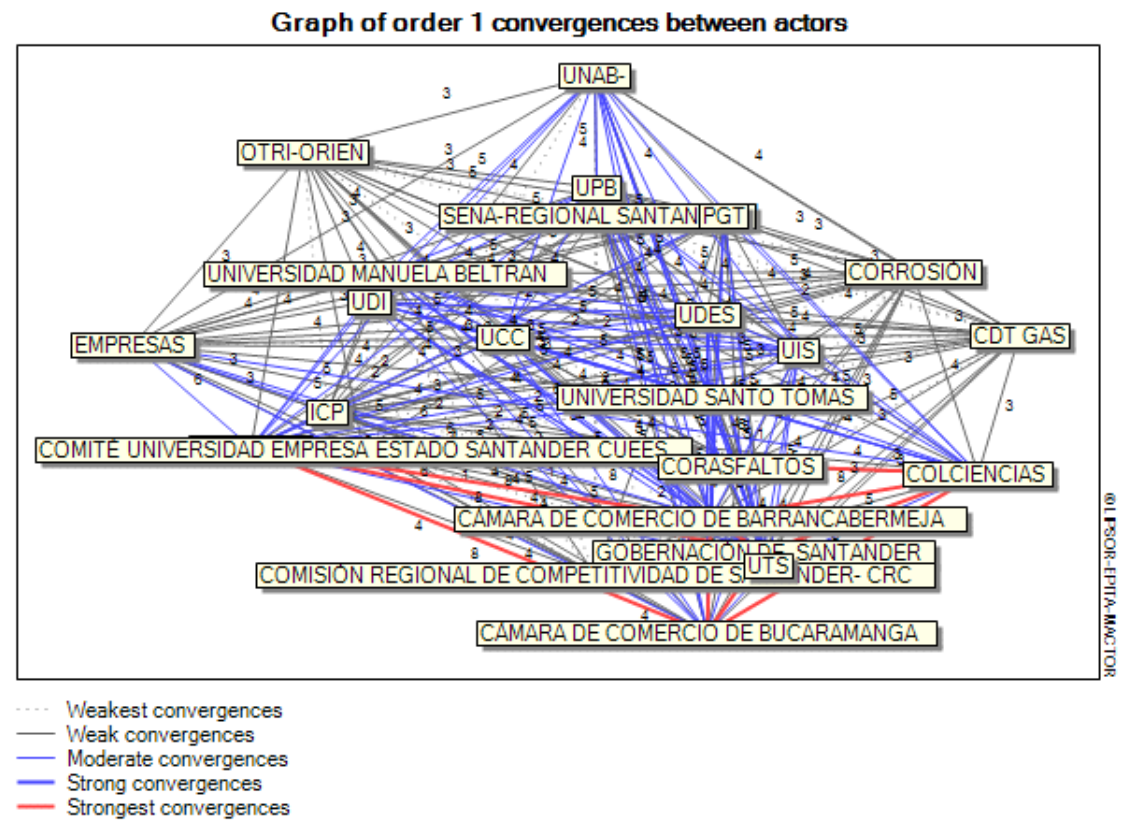

Fuente: elaboración propia usando Lipsor Mactor ${ }^{\circledR}$.

De acuerdo a la posición de los actores frente a cada una de las estrategias de articulación, el grado de compromiso de los actores por importancia es: alinear las necesidades con la oferta de servicios, fortalecer grupos y redes, cofinanciar proyectos, apoyar formación de capital humano, crear espacios de interacción, incrementar convenios de cooperación, realizar eventos de integración y financiar proyectos conjuntos. Jerarquía que difiere en gran medida de los intereses del sistema nacional de ciencia, tecnología e innovación, según los resultados obtenidos por Monroy (2006), donde la prioridad es otorgada a cofinanciar proyectos, realizar eventos de integración y financiar proyectos conjuntos. 


\subsection{Influencia de la industria del petróleo en el SRI de Santander}

La influencia de la industria del petróleo en el SRI se presenta de acuerdo a cada una de las variables clave del modelo de indicadores de CTI (Universidad Industrial de Santander, 2013), las cuales son: capital humano, producción científica, sistema de investigación y efectos económicos.

\subsubsection{Capital humano}

Entre 2001 y 2011 el número de graduados en Santander tuvo un comportamiento variable, presentando en 2005 y 2006 la población de graduados más baja en los 11 años evaluados, en los cuales tal población pasó 6.910 en 2001 a 12.985 en 2011, es decir, tuvo un incremento del 88\%. La participación de los graduados en áreas afines con petróleo en el total de formados en departamento varió entre $7,1 \%$ y 14,2\% en el mismo periodo. Igualmente, la participación de graduados de los mismos en los niveles tecnológico, universitario y especializaciones tuvo un comportamiento cambiante pero poco representativo y, una participación nula hasta el 2010 en el nivel técnico. La participación en la formación en maestrías es la segunda más representativa, que hasta 2011 había variado entre 9\% en 2001 y $48 \%$ en 2008. Mientras que la formación de doctorados no presentó grandes variaciones y en él tuvo la mayor intervención, debido a que los pocos graduados en este nivel de educación egresan, en su mayoría, en carreras afines con el petróleo. Al año 2013 el departamento tenía 20 graduados de doctorado en áreas afines con el petróleo, de los cuales ocho están directamente vinculados con organizaciones de la industria del petróleo (seis en el Instituto Colombiano del Petróleo).

\subsubsection{Producción científica}

En la Figura 2a se muestra la red de colaboración de publicación de artículos por los actores del sistema regional de innovación registrados en la base de datos ISI Web of Knowledge, los cuales eran en total 1.468 a 2013. La Universidad Industrial de Santander (UIS), el Instituto Colombiano Petróleo (ICP) y la Universidad Autónoma de Bucaramanga (UNAB) son las principales generadoras de este tipo de productos científicos en el sistema. Se observó, además, que los principales actores que publican en el departamento hacen parte del CPG. 
Figura 2 - Red de colaboración de publicación de artículos científicos
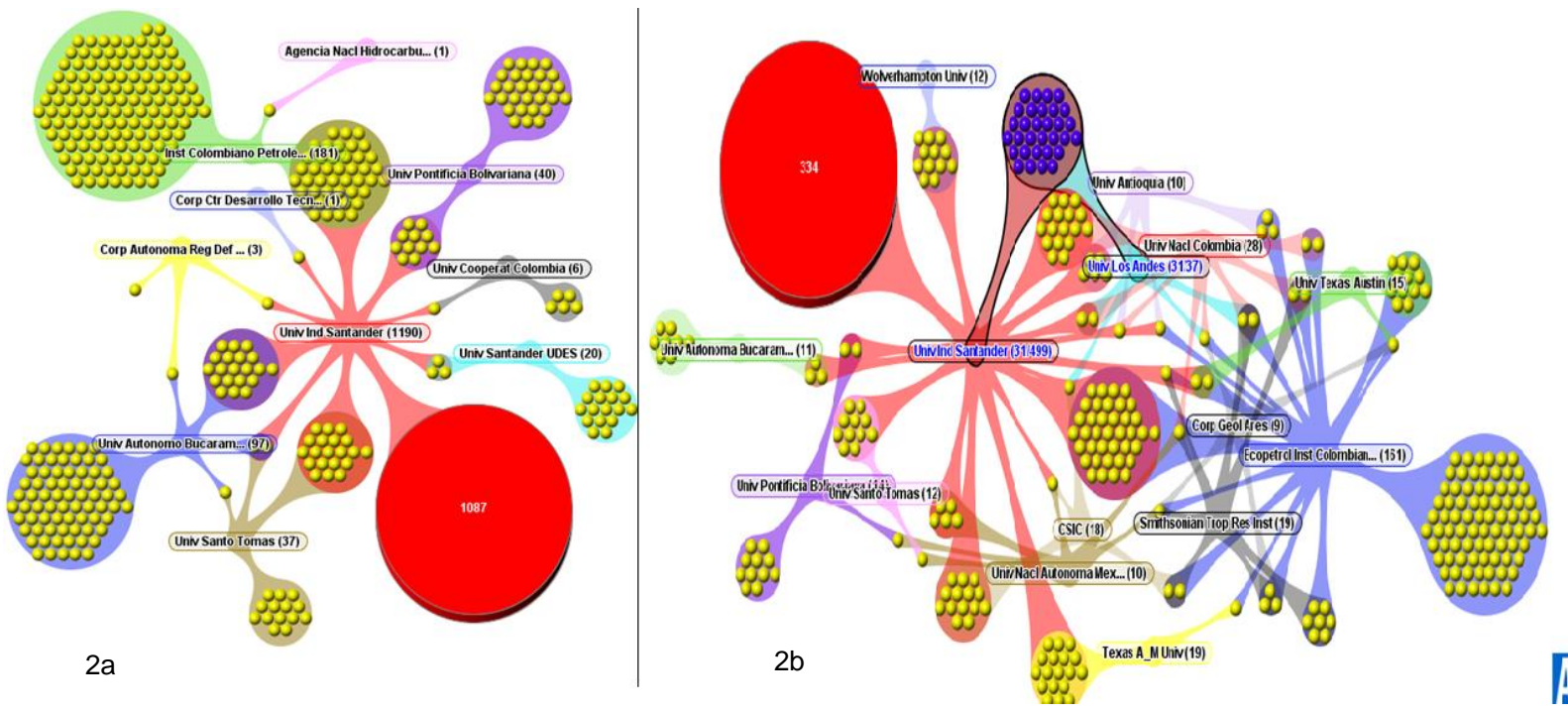

a - los diez principales del SRI de Santander $\mathrm{b}$ - del CPG en áreas de conocimiento de petróleos

Fuente: adaptada de ISI Web of Science (2014) usando Vantage Point ${ }^{\circledR}$.

A pesar de que las publicaciones son realizadas por los actores del CPG, no todas ellas se dedican al estudio de lo directamente concerniente a la industria del petróleo. Por esta razón se realizó un filtro por áreas de conocimiento para obtener una red de colaboración más aproximada a la producción científica de las áreas relacionadas con el petróleo (figura 2b); la producción total de artículos posterior a 2001 en esta red es de 661, que equivale al 45\% de total de la producción del sistema. La dinámica de publicación de éstos es similar al total del SRIS: la Universidad Industrial de Santander (UIS) y el Instituto Colombiano del Petróleo (ICP) continúan liderando la lista, siendo la Universidad de los Andes y la Universidad Nacional de Colombia los principales aliados fuera de la región; la participación anual de estas publicaciones relacionadas con petróleo posterior al 2001 fue del 41\%, lo que refleja que ésta tiene una incidencia en el comportamiento de la productividad científica regional año a año.

Los registros de patentes concedidos por la Organización Mundial de la Propiedad Intelectual (WIPO) y la Oficina de Patentes y Marcas de Estados Unidos (USPTO) a la Empresa Colombiana de Petróleos son 125 y las producidas por Corasfaltos son 9, todas agrupadas en 37 familias o invenciones. La producción de patentes ha decrecido en los últimos años, presentando su mayor pico en 2010 con 27 patentes distribuidas en 6 familias. Los sectores en los cuales han sido presentadas las patentes corresponden a: química, ingeniería mecánica, ingeniería eléctrica e 
instrumentos, siendo las dos primeras las principales. A diferencia de la red de colaboración de la producción de artículos, al escanear la red de aplicantes de patentes se evidencia vínculos con entidades de otros departamentos colombianos más no con actores de Santander.

\subsubsection{Sistema de investigación}

En Colombia, 5.500 grupos de investigación fueron reconocidos en la convocatoria de Colciencias en el año 2013. De estos, 265 se encuentran ubicados en el departamento de Santander, cifra que representa el $4.8 \%$ de total nacional y que ubica al departamento como el cuarto de la nación. De los 265 grupos de investigación de Santander, 15 están relacionados con la industria del petróleo, esto es el 5,7\% del total de grupos, los cuales están avalados por diferentes instituciones; la UIS y el ICP concentran el $80 \%$ de ellos (COLCIENCIAS, 2014).

En el país, Colciencias es la entidad encargada de evaluar la calidad de los grupos de investigación con el modelo de medición vigente, en las categorías A1, A, B, $\mathrm{C}$ y D, teniendo una valoración de A1 los grupos con mayor reconocimiento y D los de menor. Como se expone en la Figura 3, la mayor parte de los grupos del departamento, al igual que los del sector, se encuentra en la categoría C, seguida de la B. Sólo un grupo de investigación en el área, fue reconocido en la categoría A.

En Santander, para el año 2013 había 23 investigadores activos, reconocidos por Colciencias, en el área de petróleo, número que no ha presentado grandes variaciones en los últimos diez años, cuyo promedio ha sido de 21. Sin embargo, si se analiza el total de investigadores registrados en los grupos, clasificados y no clasificados por Colciencias, la tendencia del número de investigadores anuales fue creciente entre 2000 y 2009, y cayó en los años siguientes hasta 2013, donde se registran 283 integrantes de grupos. 
Figura 3 - Clasificación grupo de investigación del SRIS y del sector

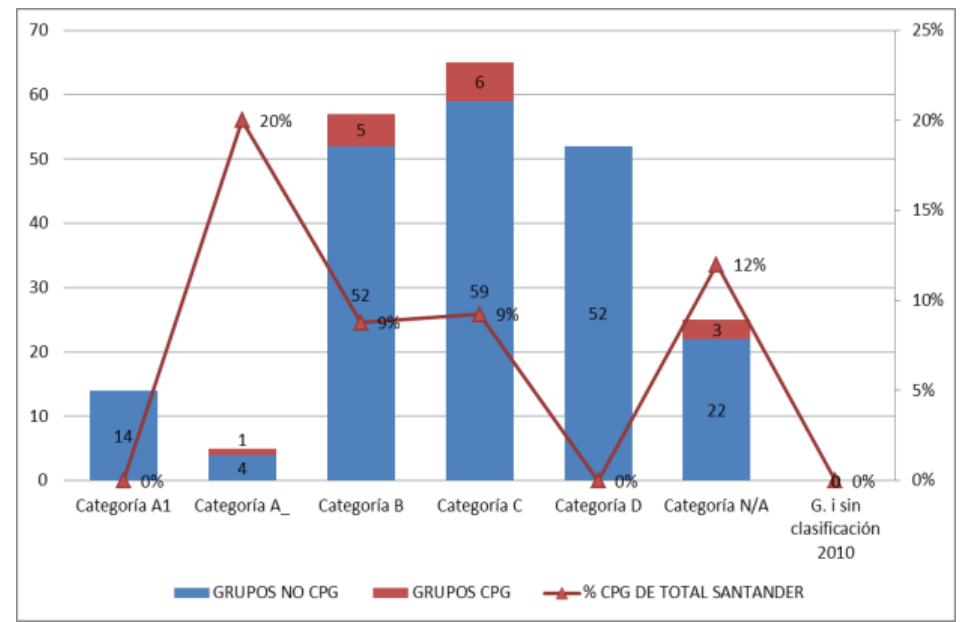

Fuente: adaptada de Colciencias (2014).

\subsubsection{Efectos económicos}

La industria en Santander se encuentra concentrada en las actividades de refinación de petróleo y en la producción de alimentos y bebidas. La Encuesta Anual Manufacturera 2013 (Departamento Admistrativo Nacional de Estadísticas de Colombia, 2014) reveló que los productos de la refinación del petróleo representan el $79,9 \%$ del total de la producción industrial de la región y el 84,1\% del valor agregado, la cual aportó el 27\% del PIB departamental en 2011 y el 22,2\% en 2012; además, la explotación del petróleo crudo y de gas natural ha tenido una participación destacada en el mismo, la cual en 2011 aportó el 6\% del total del PIB de Santander (MINISTERIO DE COMERCIO, INDUSTRIA Y TURISMO, 2014).

La participación de las importaciones de la industria del petróleo de Santander en el total de importaciones de Santander fue de 0,22\% en 2011 y 0,85\% en 2012, lo que indica que el departamento requiere poca cantidad de bienes del exterior en esta industria en comparación con los otros que importa. Mientras que las exportaciones de petróleo y sus derivados en 2012 y 2013 superaron más de la mitad de las exportaciones, 73\% en 2012 y 70,5\% en 2013 (CÁMARA DE COMERCIO DE BUCARAMANGA, 2013).

La dinámica empresarial, expresada en el número de empresas activas en el departamento, ha mostrado un crecimiento en los últimos años. Este mismo comportamiento lo han tenido las empresas de la industria del petróleo, pero a pesar del crecimiento en número, representan un mínimo porcentaje del total en la región según 
los datos registrados en las cámaras de comercio del Departamento, mientras que la cancelación de empresas en los últimos cuatro años ha sido técnicamente de cero en comparación con el total departamental, lo cual representa un buen indicador de la consolidación y supervivencia de las empresas de la industria. De otra parte, la distribución de las empresas en Santander por su tamaño no es homogénea: del total de empresas (68.714 a 15 de mayo de 2014), el 94\% eran micro, el 4\% pequeñas y, grandes y medianas el 1\% (CCB, 2014). Las empresas del sector petróleo guardan un comportamiento similar, presentando la mayor participación al en el total de empresas medianas y grandes con el 1,23 y $0,96 \%$ respectivamente.

Las empresas que conforman el clúster el petróleo y gas, aunque en su mayoría no están registradas en las cámaras de comercio del departamento en actividades propias de la industria sino en complementarias, ascienden a 446, sus áreas de actuación principales se agrupan en: Metalmecánica, Ingeniería y arquitectura, Obras Civiles y Transporte, siendo el primer área la que integra el mayor número de empresas, específicamente en la actividad de mantenimiento y reparación especializada de maquinaria y equipo con 59 empresas. Sin embargo la actividad que más empresas asociadas tiene es la de "construcción de otras obras de ingeniería civil", perteneciente al área de 'obras civiles', con 183 empresas, lo que representa al 41\% del clúster.

\subsection{La influencia de la industria del petróleo en la industria de asfaltos y pavimentos}

Desde el punto de vista económico, la industria de asfaltos y pavimentos es influenciada por la industria del petróleo, principalmente porque el inicio de la cadena de la segunda es alimentada por la culminación de la primera. En el caso del Departamento de Santander, la industria de asfaltos y pavimentos tiene una fuerte presencia, lo cual se explica porque en él se ubica la refinería de mayor capacidad de Colombia. Dada la sabida influencia económica, a continuación se expone los factores que justifican la existencia de una influencia científico-tecnológica.

\subsection{Formación de recursos humanos}

El número de proyectos de grado de los egresados de educación superior entre 2000 y 2013 relacionados con el estudio de asfaltos presentó un comportamiento variable, con un máximo en 2010. De estos proyectos, los graduados en programas 
afines con la industria del petróleo, entre 2000 y 2012, tuvieron una participación que varió entre el $40 \%$ y el $60 \%$ de los mismos, lo cual indica que estos programas relacionados con petróleo proporcionan una plataforma para la formación de capital humano en asfaltos. El porcentaje restante de los mismos fueron desarrollados en su mayoría en el área de conocimiento de la ingeniería civil y el mayor aporte lo realizó la UIS con 113 proyectos de entre 2000 y 2013.

Los investigadores en asfaltos de los grupos relacionados con petróleo son, en número, muy similar tanto para los reconocidos por Colciencias como para los no reconocidos, lo cual indica una buena la calidad de los investigadores y por ende de los trabajos que estos desarrollan. Además, se evidencia que la participación de éstos investigadores en asfaltos de la industria del petróleo, según la categorización de Colciencias varió entre el 25\% y 65\% entre los años 2000 y 2013, teniendo la media de participación en el $43 \%$.

\subsubsection{Registro y homologación de patentes}

De las patentes producidas por los actores de la industria del petróleo, 13 están relacionadas con asfaltos; los dos aplicantes de éstas son Corasfaltos y Ecopetrol, quienes no presentan trabajos conjuntos en la producción de las mismas.

\subsubsection{Consolidación de capacidades para realizar actividades de I\&D}

De los quince grupos de investigación en petróleos, seis han realizado investigaciones o presentan líneas de investigación en asfaltos (Figura 4a), los cuales están avalados por tres entidades: Instituto Colombiano del Petróleo, UIS y Corasfaltos, el cual nace también de la iniciativa de algunos actores entre los que están los dos primeros. 
Figura 4 - Grupos de investigación y red de colaboración
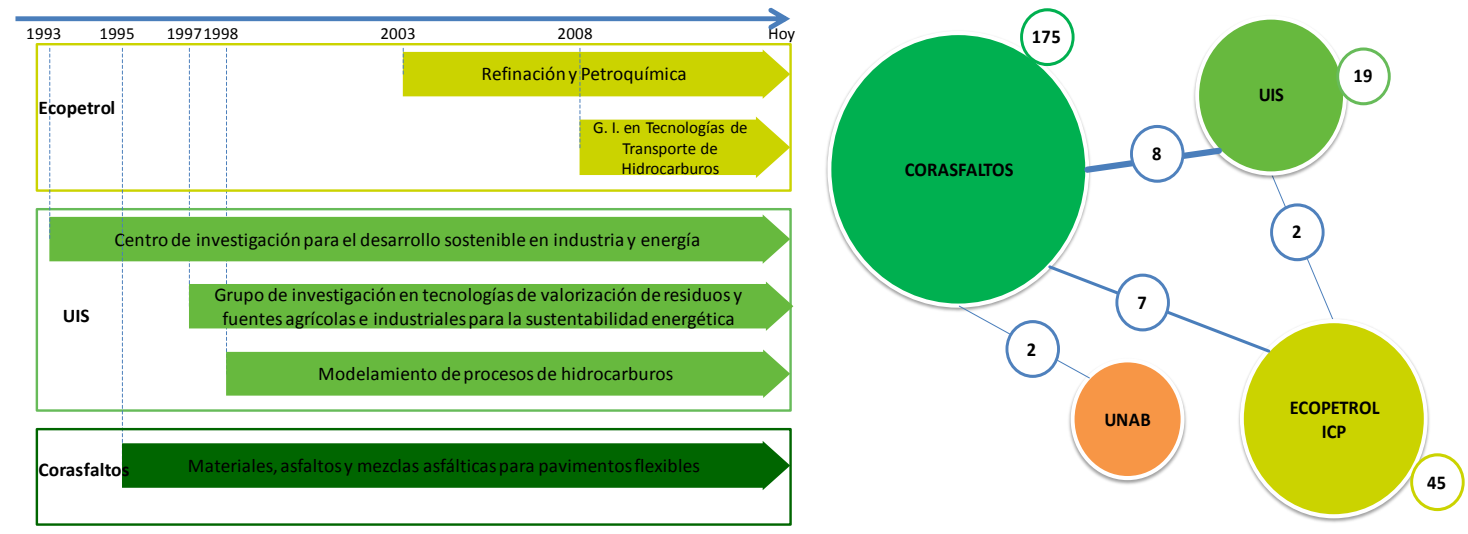

a - Grupos de investigación relacionados con asfaltos. b - Red de cooperación de productos científicos.

Fuente: adaptada de Colciencias (2014).

También se encontró que la entidad relacionada con la industria del petróleo que mayor número de productos tiene de forma individual en asfaltos es Corasfaltos, seguido del ICP (Figura 4b). La mayor cooperación del primero se presenta con la UIS, seguido del IPC y finalmente con la UNAB, por proyectos de grado de pregrado.

De los laboratorios de los actores del clúster del petróleo ocho, avalados por tres entidades, podrían realizar actividades relacionadas con asfaltos. Nuevamente, las entidades corresponden a la UIS, CORASFALTOS e ICP. Además, es importante hacer mención que el fortalecimiento de los laboratorios de Corasfaltos fue apoyado la UIS y el ICP.

\subsubsection{Publicaciones, cooperación regional e internacional}

Los artículos científicos relacionados con asfaltos, en su mayoría, han sido producidos por la UIS y el ICP en la misma proporción entre 2009 y el primer trimestre de 2014. También se encuentran artículos publicados por Corasfaltos y la empresa CPT, en una menor cantidad. La Figura 5a muestra que la producción de estos ha tenido un comportamiento variable, con una producción casi nula antes de 2008 y con su mayor pico en 2012. Como se muestra en la red de cooperación en la Figura 5b, estas entidades han producido artículos conjuntos, exceptuando a Corasfaltos, cuya cooperación es con entidades ubicadas fuera del país. De la red, se observa que sólo una pequeña proporción de las relaciones se presenta con los actores del sistema nacional y regional de innovación. La cooperación internacional de las publicaciones se ha realizado con 
entidades ubicadas en Canadá, España, Brasil, Venezuela, Francia, México, Suráfrica y Estados Unidos.

Figura 5 - Artículos publicados por los actores del SRI de Santander
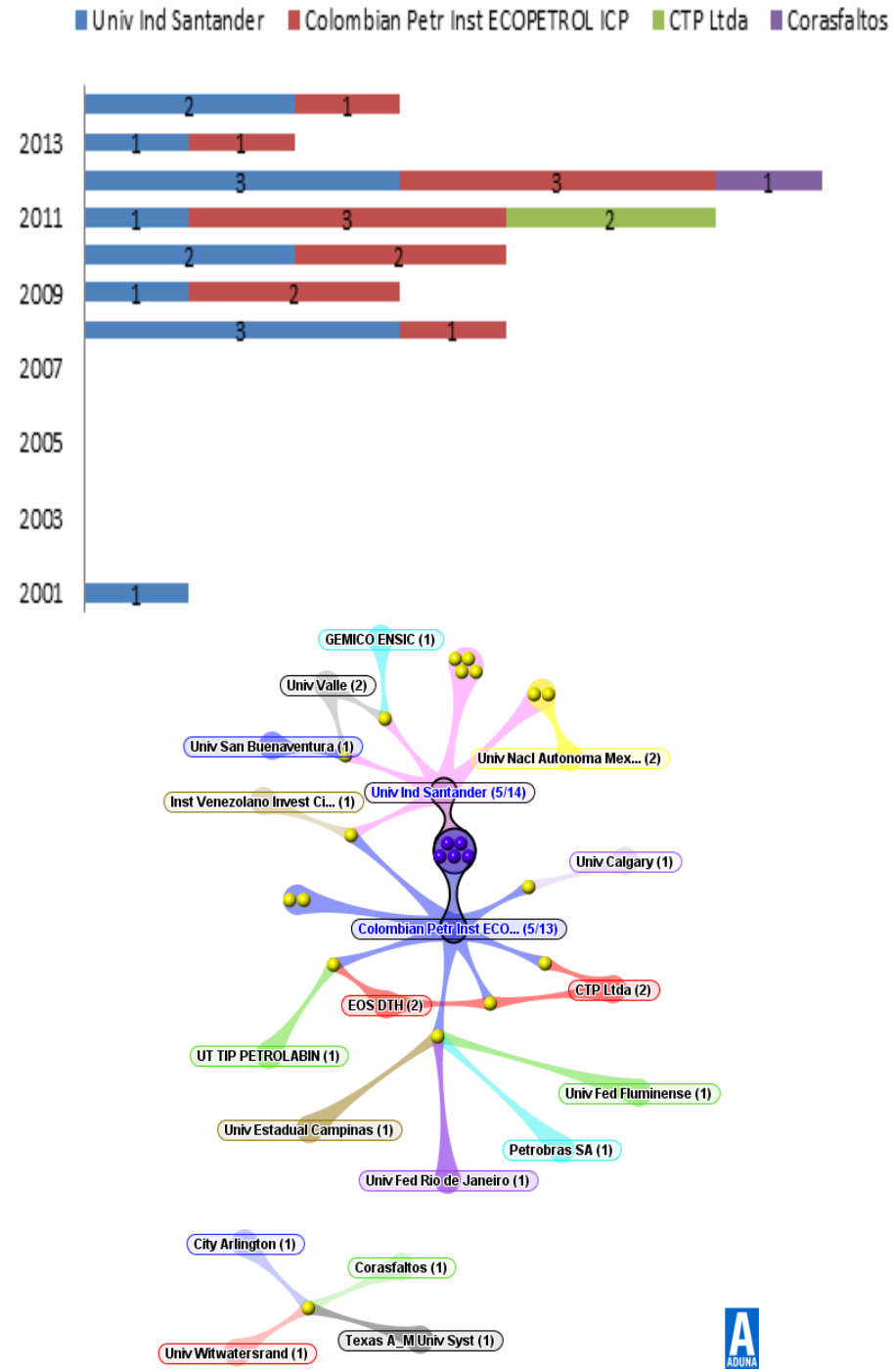

$\mathrm{a}$ - publicaciones por años.

$\mathrm{b}$ - red de colaboración.

Fuente: adaptada de ISI Web of Knowledge (2014).

En las variables "Mejoramiento en la oferta y pertinencia de los servicios tecnológicos" y "Desarrollo de capacidades de diseño e ingeniería", "Registro y documentación técnica del know-how", no se encontró evidencia de influencia directa de la industria del petróleo en la de asfaltos y pavimento.

\section{Conclusiones}

Los actores del sistema regional de innovación del departamento de Santander que pertenecen al clúster del petróleo y gas son el 32\% del mismo, en el cual hay una 
fuerte presencia de los agentes de los subsistemas científico- académico con un $67 \%$ de participación. El 76,5\% de los actores pertenecen del sector privado, que al igual que el público, es liderado por entidades científico-académicas. El $79 \%$ de las entidades corresponden a organizaciones, es decir, a "jugadoras del juego", y sólo el 21\% son instituciones, lo que indica una buena conformación del sistema en el área de petróleo, ya que las segundas tienen funciones de soporte a la innovación y las organizaciones tienen el papel principal en el mismo. De otra parte, en el entramado de actores sólo el $70 \%$ de ellos establecen relaciones significativas, la mayor parte de ese restante $30 \%$ corresponden a instituciones universitarias del subsistema científico-académico. Los actores del sistema facilitador presentan las relaciones más fuertes, lo cual se explica porque todos ellos cumplen funciones similares para garantizar una plataforma de innovación a los restantes agentes o por una debilidad del sistema, identificada en otros estudios, que es el traslape de funciones en este subsistema.

La mayor participación de la industria en la formación de capital humano se presenta en el nivel de doctorado y maestría, lo que es explicado por la escasa cantidad de graduados en estos niveles de formación a nivel departamental y a que la mayoría de ellos son egresados de programas relacionados con la industria del petróleo. De igual forma, la mayor parte de los artículos científicos producidos por los agentes del sistema regional de innovación encontrados en la base de datos fueron presentados por organizaciones del clúster del petróleo y gas, de los cuales el $45 \%$ están relacionadas con petróleo. Así mismo, la gran mayoría de las patentes fueron producidas por actores del clúster, las cuales se agrupan en 37 familias en las áreas de química, ingeniería mecánica, ingeniería eléctrica e instrumentos, en colaboración con entidades de otros departamentos colombianos más no con actores del SRI de Santander.

Los grupos de investigación con líneas relacionadas con petróleo son el 6\% del total de grupos de la región, de los cuales el $80 \%$ están avalados por la UIS y el ICP y el $59 \%$ de ellos presentaron una calidad media según la clasificación realizada por Colciencias en 2013. Desde el punto de vista empresarial, el número de empresas activas en el sector ha mostrado un bajo crecimiento frente a otros sectores, sin embargo la mortalidad de las mismas ha sido casi nula en los últimos años, el mayor aporte en número de la industria al sistema por tamaño empresarial se presenta en la de empresas medianas y grandes. 
Además de la conocida influencia económica de la industria del petróleo en la de asfaltos y pavimentos, se comprobó una relación científico-tecnológica, la cual se hizo evidente en variables como formación de recurso humano porque los graduados en carreras afines con petróleo realizaron proyectos de grado relacionados con asfaltos y porque el número de investigadores asociados a los grupos de investigación de esos programas investigan en asfaltos, los cuales, en una buena proporción están clasificados por Colciencias. Así mismo, en producción de patentes y artículos científicos también se observó una influencia, con énfasis en la producción de artículos, donde la tendencia del total producido en la región es similar a la del clúster del petróleo y gas, representando un porcentaje significativo del mismo. Esta influencia puede ser explicada porque organizaciones y grupos de investigación en petróleo adelantan estudios en asfaltos. Las principales instituciones participantes son Corasfaltos, el Instituto Colombiano de Petróleo y la Universidad Industrial de Santander, que tienen la mayor cantidad de productos científico-tecnológicos entre sí, en comparación con los que tienen con entidades fuera de la región.

A lo largo del estudio se evidenció la participación del Instituto Colombiano del Petróleo y Universidad Industrial de Santander como dos de los principales actores del clúster que han dado un aporte significativo al desarrollo del Sistema Regional de Innovación de Santander. Instituciones que han venido trabajando juntas desde la misma creación del ICP, adelantando investigaciones que han resultado en diferentes productos científicos y tecnológicos, como artículos, prototipos y patentes, evidenciando un caso de éxito de la vinculación universidad-empresa.

\section{REFERENCIAS}

ARIAS, C.; ARENAS, P. Diagnóstico en ciencia, tecnología e innovación en el Departamento de Santander. 2013. Tesis de grado - Universidada Industrial de Santander Bucaramanga, 2013.

ARIAS, C.; ARENAS, P.; FLÓREZ, Y.; CARRILLO, E. Una aproximación al sistema regional de innovación del Departamento de Santander. Revista Gerenc. Tecnol. Inform, p. 45-58, 2013.

ASHEIM, B. T.; ISAKSEN, A. (). Location, agglomeration and innovation: Towards regional innovation systems in Norway? STEP Report, 1996. 
Regional Innovation Systems: The Integration of Local "Sticky" and Global “Ubiquitous” Knowledge. Journal of Technology Transfer, v. 27, p. 77-86, 2002.

ASHEIM, B. T.; LAWTON, H.; OUGHTON, C. Regional Innovation Systems: Theory, Empirics and Policy. Regional Studies, v. 45, n. 7, p. 875-891, 2011.

AUTIO, E. Evaluation of RTD in regional systems of innovation. European Planning Studies, v. 6, n. 2, p. 131-140, 1998.

BRESCHI, S. The geography of innovation: a cross-industry analysis. Regional Studies, v. 34, p. 213-229, 2000.

CÁMARA DE COMERCIO DE BUCARAMANGA. Santander Exporta, n. 85, 2013.

COOKE, P. Planet Europe: Network Approaches to Regional Innovation and Technology Management. Technology Management, v. 2, p. 18-30, 1995.

COOKE, P.; BOEKHOLT, P.; TÖDTLING, F. The Governance of Innovation in Europe. London: Cengage Learning, 2000.

COOKE, P.; ROPEER, S.; WYLIE, P. The Golden Thread of Innovation and Northern Ireland's Evolving Regional Innovation System. Regional Studies, v. 37, n. 4, p. 365$379,2003$.

COOKE, P.; URANGA, M. G.; ETXEBARRÍA, G. Regional innovation systems: Institutional and organizational dimensions. Research Policy, v. 26, p. 475-491, 1997.

DE LUCIO, I. F.; CASTRO, E. La nueva política de articulación del sistema de innovación en España. In: VI Seminario Latino-iberoamericano de Gestión Tecnológica, ALTEC, 1995. Concepción, Chile, 1995.

DEN HERTOG, P.; ROELANDT, T.; BOEKHOLT, P. Assesing the Distribution Power of National Innovation Systems Pilot Study: The Netherlands. Apeldoorn, OECD, 1995.

DEPARTAMENTO ADMINISTRATIVO NACIONAL DE ESTADÍSTICAS DE COLOMBIA. La Encuesta Anual Manufacturera 2013. Bogotá, 2014.

DOLOREUX, D. What we should know about regional systems of innovation. Technology in Society, v. 24, p. 243-263, 2002. 
DOLOREUX, D.; PARTO, S. Regional innovation systems: Current discourse and unresolved issues. Technology in Society, v. 27, n. 2, p. 133-153, 2005.

EDQUIST, C. Systems of Innovation: Technologies, Institutions, and Organizations. Psychology Press, 1997.

FURMAN, J.; PORTER, M.; STERN, S. The determinants of national innovative capacity. Research Policy, v. 31, p. 899-933, 2002.

HOWELLS, J. Regional systems of innovation? In: ARCHIBUGI, D.; HOWELLS, J.; MICHIE, J. Innovation Policy in a Global Economy. Cambridge: Cambridge University Press, 1999. p. 67-93.

IAMMARINO, S. An evolutionary integrated view of Regional Systems of Innovation: Concepts, measures and historical perspectives. European Planning Studies, v. 13, n. 4, p. 497-519, 2005.

INZELT, A. The evolution of university-industry-government relationships during transition. Research Policy, v. 33, p. 975-995, 2004.

ITURRIAGAGOITIA, J. Z.; SAEZ, F. J.; GRACIA, A. G. Innovation systems: need to define new network. In: International conference on engineering design iced $\mathbf{0 5}$ Melbourne. Melbourne, 2005. p. 1-10.

LANGFORD, C. Academy of Management Conference Proceedings. Confronting Complexity in Technology Cluster Development: Towards an Evolutionary Theory. Philadelphia, 2007.

LI, Z. Research on Evaluation Index System of Regional Innovation. Management Science and Industrial Engineering (MSIE), p. 1269-1272, 2011.

MENÉNDEZ, A.; DE LUCIO, I. F.; JIMÉNEZ, F. Sistemas Regionales de Innovación. Caracterización y situación en América Latina. In: Los sistemas Regionales de innovación en América Latina, p. 19-43, 2011.

MINISTERIO DE COMERCIO, INDUSTRIA Y TURISMO. Caracterización económica del Departamento de Santander. Bogotá, 2014. 
MONROY, S. Nuevas políticas y estrategias de articulación del sistema de ciencia, tecnología e innovación colombiano. Innovar, v. 16, n. 28, p. 157-172, 2006.

MORGAN, K. The exaggerated death of geography: learning, proximity and territorial innovation systems. Journal of Economic Geography, v. 4, p. 3-21, 2004.

SARMIENTO, L.; JAIME, A.; ARENAS, P.; BECERRA, A.; CAMACHO, J. Metodología para la evaluación de impactos de proyectos de I+D. Caso de aplicación: financiación COLCIENCIAS 1999-2005. En temas de Indicadores de Ciencia y tecnología. Argentina: Red Iberoamericana De Indicadores De Ciencia Y Tecnologia Ricyt, 2011.

TÖDTLING, F.; TRIPPL, M. One size fits all? Towards a differentiated regional innovation policy approach. Research Policy, v. 34, n., p. 1203-1219, 2005.

TRIPPL, M.; TÖDTLING, F. Developing Biotechnology Clusters in Non-high Technology Regions - The Case of Austria. Industry \& Innovation, v. 14, n. 1, p. 4767, 2007.

UNIVERSIDAD INDUSTRIAL DE SANTANDER. Plan estratégico de ciencia, tecnología e innovación del Departamento de Santander. Bucaramanga, 2013.

WANGNER-DÖBLER, W. The system of research and development indicators: Entry points for information agents. Scientometrics, v. 62, p. 145-153, 2005.

YUN, S.; LEE, J. An innovation network analysis of science clusters in South Korea and Taiwan. Asian Journal of Technology Innovation, v. 21, n. 2, p. 277-289, 2013. 